\title{
MULHERES, TEMPOS E ESPAÇOS NA CIÊNCIA AGROPECUÁRIA PAULISTA'
}

\author{
WOMEN, TIME AND SPACE IN AGRICULTURAL SCIENCE OF SÃO PAULO STATE, BRAZIL
}

\begin{abstract}
RESUMO
Este artigo olha para mulheres de diferentes tempos e a conquista por espaços na ciência agropecuária paulista. Neste sentido, tem como objetivo principal verificar a relação entre atributos sociodemográficos e o desenvolvimento da carreira de pesquisadoras lotadas em instituições públicas de pesquisa paulistas, no contexto do agronegócio. Para tanto, fez-se uso do aporte teórico-metodológico da demografia organizacional e dos estudos de gênero e geração nas ciências. $\mathrm{O}$ banco de dados sociodemográfico foi concedido pela unidade gestora das instituições e a produção bibliográfica extraída dos currículos cadastrados na Plataforma Lattes (CNPq). Em seguida, a partir de uma abordagem quantitativo-descritiva, foram realizadas análises estatísticas uni e multivariadas. Os resultados permitiram descrever a participação, contribuição e inserção feminina no contexto investigado. A partir disso, observou-se a complexidade do fenômeno, no qual a tríplice linha argumentativa qualificaçãomaternidade-produtividade mostrou-se insuficiente para explicar a baixa penetração feminina nos mais altos cargos de direção.
\end{abstract}

Palavras-chave: Mulheres na ciência agropecuária. Gênero. Geração.

\begin{abstract}
This article looks at women from different periods of time and her conquest of spaces in the agricultural science of São Paulo State, Brazil. In this sense, the purpose is to verify the relationship between sociodemographic attributes and the career development of women scientists at research institutions in São Paulo State (agribusiness), Brazil. For this purpose, the theoretical and methodological contribution of the Organizational Demography and Gender and Generation in Science' studies was used. Sociodemographic database was provided by research institutions and bibliographic production was extracted directly from Lattes Platform $(\mathrm{CNPq})$. It was a quantitativedescriptive approach with univariate and multivariate statistical analyses. The

1 Uma versão preliminar deste artigo foi apresentada no Simpósio Temático (ST) "A mulher no mundo da Ciência e do Trabalho: permanências e transformações" do Seminário Internacional Fazendo Gênero $11 \& 13^{\text {th }}$ Women's Worlds Congress (FG11 \& WWC, 2017) e, posteriormente, publicada nos anais eletrônicos do congresso. Esta versão incorpora as contribuições dos coordenadores do ST, Dra. Isabel Cruz Lousada (Universidade Nova de Lisboa) e Dr. Márcio Matiassi Cantarin (Universidade Tecnológica Federal do Paraná), bem como das/os demais pesquisadoras/es presentes. Aproveitamos para agradecer ao amigo e cientista da computação Roberto Hayasida Junior pelo apoio na extração dos dados na Plataforma Lattes (CNPq). Por fim, aos apontamentos realizados no parecer deste Periódico.
\end{abstract}

Kris H. Oliveira

Universidade Estadual de Campinas (Unicamp). E-mail: kris.h.oliveira@gmail.com

Márcia M. P. Serra

Universidade Presbiteriana Mackenzie. E-mail: mapiserra@gmail.com 
results describe the women's participation, contribution and insertion in the context investigated. In addition, it was observed the complexity of the phenomenon, in which the triple argumentative line qualification-maternity-productivity was insufficient to explain the low female penetration in higher management positions.

Keywords: Women in agricultural science. Gender. Generation.

\section{Introdução}

A partir da segunda metade do século $\mathrm{XX}$, mulheres cientistas passaram a questionar mais intensamente uma realidade social que as distanciava da carreira científica. Tempos de enunciação feminina em espaços que se reivindicava transbordar. Tamanha contestação ganhou fôlego com o apoio do movimento feminista e estimulou a produção de pesquisas sobre a participação feminina nas ciências. Frente ao desejo e às estatísticas, governos e instituições de ensino e pesquisa têm se visto pressionados a responder com medidas pontuais em prol da não discriminação e equidade de gênero na formação, áreas e carreiras relacionadas aos campos da ciência e tecnologia.

Apesar das transformações sociais e institucionais, estudos têm evidenciado que as desigualdades de gênero no campo científico ainda são latentes. E, sendo assim, há muitas questões em aberto sobre a participação, contribuição e inserção feminina nas ciências que não têm recebido atenção necessária da pesquisa social (Leta, 2014; Costa \& Feltrin, 2016).

O agronegócio e as ciências agropecuárias são exemplos de áreas masculinizadas. As instituições públicas de pesquisa inseridas neste campo são de grande importância nacional, porque dentre os setores econômicos brasileiros este é um dos três que mais concentram profissionais no país (IBGE, 2012) trabalhando, em sua maioria, no estado de São Paulo (CGEE, 2012). Ademais, destaca-se como o setor econômico que mais cresceu na última década (Brasil, 2011).

Este estudo tem como objetivo verificar a relação entre atributos sociodemográficos e o desenvolvimento da carreira de pesquisadoras lotadas em instituições públicas de pesquisa paulistas, no contexto do agronegócio. Especificamente, busca: i) descrever o perfil sociodemográfico e de produção bibliográfica das pesquisadoras, em comparação com os homens e grupos etários; ii) verificar a relação entre idade, nupcialidade, maternidade, produção bibliográfica e a ocupação de altos cargos nas instituições de pesquisa; iii) refletir sobre as transformações e estagnações na relação com a abordagem de gênero e geração nas ciências.

Pesquisas empíricas sobre as cientistas atuantes em instituições públicas de pesquisa, inseridas num contexto de mudanças demográficas e socioculturais, ainda são incipientes. Isto se justifica, pois, informações estatísticas sobre estas instituições no Brasil ainda se encontram dispersas e de difícil acesso (Leta, 2003). 
A relação entre atributos sociodemográficos e o desenvolvimento da carreira de pesquisadoras também são questões pouco exploradas, principalmente no Brasil (Oliveira et al., 1999). Reflexões sobre as estatísticas na relação com a abordagem de gênero e geração nas ciências mostram-se ainda mais embrionárias.

Este artigo realiza, portanto, uma leitura acerca da participação, contribuição e inserção feminina em um campo onde as mulheres são historicamente subrepresentadas: a ciência agropecuária. A seguir, será apresentado um breve apanhado teórico sobreos estudos de gênero egeração nas ciências, bem como sublinhadas as suas lacunas. Em seguida, descritos os procedimentos metodológicos da pesquisa. Na seção de análise dos dados, especial destaque será dado às características sociodemográficas, à produção bibliográfica e ao desenvolvimento da carreira das pesquisadoras. Por fim, serão apresentadas as conclusões alcançadas diante dos objetivos propostos.

\section{Gênero, Geração e Ciência}

De acordo com Scott (1995), pensar sobre gênero requer olhar para o modo como se percepcionam as diferenças sexuais em diferentes contextos, o qual se atribui um modo de se pensar hierarquizado e dual. Logo, quando o sexo é entendido enquanto uma matéria social este, por sua vez, não pode ser estudado de maneira dissociada do gênero. Essa teia de significados culturais, segundo a autora, está articulada a mecanismos de poder constituídos historicamente. Deste modo, as diferenças dos sexos se tornaram uma base classificatória que atribui a corpos de homens e de mulheres modos de ser, falar, trabalhar e pensar.

Neste sentido, a perspectiva de Bourdieu (2002) sobre as relações de poder contribui com a reflexão sobre o modo como aspectos masculinos e femininos permeiam a vida social cotidiana de forma assimétrica e hierárquica. Em determinado contexto, há configurações simbólicas passadas por gerações e reforçadas por instituições sociais (como a escola, a família, Igreja e o Estado) de modo a serem apreendidas como "naturais" - conforme o conceito de habitus do autor. A ciência, por exemplo, seria um campo naturalizador das desigualdades entre os sexos. Assim, quando há a supervalorização e legitimação daquilo que é entendido como masculino em detrimento do feminino expressa-se uma forma de "violência simbólica". Este conjunto de significações, por sua vez, embora com nuances em diferentes culturas, desenha esquemas de pensamento, aspirações profissionais e trajetórias individuais. Dentre elas, estão aquelas relacionadas aos campos da ciência e tecnologia.

Os primeiros estudos sobre mulheres nas ciências surgiram nos anos de 1960 . Neles, eram questionadas as restrições sociais sobre a participação de mulheres no campo científico. Com a consolidação do movimento feminista, o debate veio por inserir-se nos estudos de gênero (Leta, 2014).

Nas décadas de 1970 e 8o, as críticas de Evelyn Fox Keller ao campo científico uniram-seao movimento político feminista, cuja uma das intenções era a de transformar 
o lugar da mulher no mundo do trabalho e da ciência. Seu esforço naquele momento foi o de problematizar o status de neutralidade, objetividade e universalidade atribuídos ao fazer científico e atribuir destaque aos sujeitos que produziam a ciência. Em particular, Keller (2006) questionou a divisão mente, razão e objetividade como atributos entendidos como "masculinos", e coração (e corpo), sentimento e subjetividade como "femininos". Esta divisão, a seu ver, estaria subjacente à exclusão das mulheres nas ciências. O modo como a ciência se organiza e constitui, portanto, estaria repleta de sentidos e ações "generificadas".

No cerne desta discussão, a autora buscou sublinhar ainda as contribuições que mulheres cientistas têm oferecido para se repensar teorias clássicas, como no caso da biologia. Desta maneira, ao colocar em suspensão os eixos fundamentais da produção epistemológica e ao mesmo tempo social buscou uma ciência mais acessível às mulheres e, sobretudo, mais equânime. A partir de então, esforços passaram a ser direcionados aos estudos de gênero e ciência, projeto abraçado por outras autoras nos anos subsequentes. Todavia, o impacto do feminismo na ciência não se reservou à maior participação feminina e a equidade de direitos em relação aos homens, mas visou contemplar um novo modo de se pensar o desenvolvimento das ciências sob o espectro do masculino (Keller, 2006).

Nos anos de 1990, os estudos sobre as intersecções entre gênero, ciências e tecnologias se consolidaram no Brasil (Costa \& Feltrin, 2016). Pesquisas também foram impulsionadas por incentivos de órgãos nacionais e internacionais. A UNESCO (Organização das Nações Unidas para a Educação, a Ciência e a Cultura), por exemplo, estimulou estudos e conferências com o intuito de discutir e propor ações para a maior inclusão das mulheres nas atividades de ciência e tecnologia (Leta et al., 2006).

As alterações na dinâmica de gênero nas ciências ocorreram de maneira mais acentuada no país a partir dos anos 200o. Isto tem sido observado por meio de uma produção crescente de estudos nacionais, os quais envolvem discussões sobre: métricas de produtividade e participação feminina; áreas e carreiras típicas; segregação horizontal e vertical; cultura e sociedade; normas e legislação; discriminação; níveis de investimento às cientistas; conflitos família-trabalho (Velho \& Leon, 1998; Leta, 2003; Leta et al., 2006; Melo \& Oliveira, 2006; Moreira \& Velho, 2010; Olinto, 2011; Prado \& Fleith, 2012; Rigolin et al., 2013; Saboya, 2013; Leta, 2014; Costa \& Feltrin, 2016; Rodrigues \& Guimarães, 2016).

Por meio de uma sintética revisão da literatura, até o momento foram apresentados alguns pontos de ruptura e resistência feminista no campo científico identificados no decorrer de quatro décadas (1960-200o). No entanto, foi somente nos últimos anos que estudos nacionais passaram a contemplar as questões de gênero e geração nas ciências.

O estudo de Carvalho et al. (2013) pode ser circulado com um dos poucos a abordar tais questões de modo interseccional. As autoras entrevistaram mulheres docentes de distintas gerações (nascidas nas décadas de 1950/60 e 1970/80) lotadas em uma universidade pública brasileira, cujas áreas de atuação são consideradas masculinas (Engenharia Mecânica, Física, Matemática e Informática). Observaram 
que as duas gerações apresentam trajetórias marcadamente distintas no que diz respeito aos temas de família e carreira.

Isto posto, pôde-se notar que a "dominação masculina" no campo da ciência e tecnologia tem sido questionada enfaticamente desde a segunda metade do século XX. O movimento feminista contemporâneo mudou a percepção e a posição das mulheres na ciência, seja em número de doutoras tituladas ou na ocupação de cargos de prestígio, como de professoras titulares em universidades. Soma-se a isso o modo como a participação das cientistas em espaços historicamente masculinos tem ajudado a erodir rótulos tradicionais de gênero no campo científico (Keller, 2006).

Todavia, apesar dos desdobramentos sociais e nos estudos de gênero e ciências, a literatura tem sido pouco sensível em constatar que as cientistas podem ser de temporalidades distintas. Sendo assim, uma abordagem geracional possibilitaria observar as estagnações e transformações de gênero nas ciências e instituições de pesquisa ao longo do tempo.

\section{Procedimentos metodológicos}

Esta pesquisa configura-se como de abordagem quantitativo-descritiva, cujo aporte teórico-metodológico advém da demografia organizacional ${ }^{2}$ e dos estudos de gênero e geração nas ciências.

Para atender aos objetivos expostos na introdução deste artigo, foram adotadas 5 etapas: i) levantamento e revisão da literatura; ii) contato com a unidade gestora das instituições de pesquisa (DRH) para a coleta dos dados; iii) coleta dos currículos cadastrados na Plataforma Lattes (CNPq); iv) tratamento e construção do banco de dados sistemático com todas as variáveis - sociodemográficas e de produção bibliográfica - dos/as pesquisadores/as ( $\mathrm{N=696);} \mathrm{v)} \mathrm{análises} \mathrm{estatísticas} \mathrm{e} \mathrm{discussão}$ dos resultados. Os dados foram trabalhados pelos autores de 2013 a 2017.

As variáveis sociodemográficas são: data de admissão; nível do cargo; sexo; escolaridade; cargo de direção; data de nascimento; estado civil; número de filhos. Já as variáveis de produção bibliográfica envolvem: artigos completos publicados em periódicos; trabalhos completos publicados em anais de congressos; livros publicados/ organizados ou edições; capítulos de livros publicados; textos em jornais de notícias/ revistas.

Neste estudo, foi adaptado um indicador demográfico, a taxa média geométrica de crescimento populacional, para que fosse possível observar e comparar a evolução da produção bibliográfica das/os pesquisadoras/es. Na demografia, as estimativas de crescimento de uma população podem ser realizadas pelo método geométrico. Em termos técnicos, para se obter a taxa média geométrica de crescimento $\left(r_{g}\right)$, subtrai-

2 Segundo Pfeffer (1985), a demografia organizacional nada mais é que descrever uma organização em termos de distribuição em diversas dimensões, tais como sexo, idade, tempo de serviços prestados entre outras. Esta linha avança na aplicação de teorias específicas sobre as causas e consequências dos fenômenos demográficos para as organizações. 
se 1 da raiz enésima do quociente entre a população final e a população no começo do período considerado, multiplicando-se o resultado por 100, sendo " $t$ " igual ao número de anos no período (Cerqueira \& Givisiez, 2004). Essa lógica matemática foi adaptada ao estudo da produção bibliográfica dos pesquisadores ao longo do tempo. Desta forma, a taxa média geométrica de crescimento anual da produção bibliográfica é dada pela seguinte equação:

$$
r_{g}=\left[\left(\sqrt[t]{\frac{P B_{\text {final }}}{P B_{\text {inicial }}}}\right)-1\right] \times 100
$$

Onde:

$$
\begin{aligned}
& r_{g}=\text { taxa média geométrica de crescimento anual da produção bibliográfica; } \\
& \mathrm{I}=\text { tempo transcorrido entre as duas datas de referência; } \\
& P B_{\text {final }}=\text { volume de produção bibliográfica no fim do período; } \\
& P B_{\text {inicial }}=\text { volume de produção bibliográfica no começo do período. }
\end{aligned}
$$

As técnicas estatísticas uni e multivariadas utilizadas foram respectivamente qui-quadrado e modelos de regressão logística. A primeira comparou cada variável com a produção total. A segunda comparou as variáveis com as chances de estar em alto cargo, por total de pesquisadores e por sexo. $\mathrm{O}$ teste qui-quadrado verifica se as variáveis são independentes (não associadas) ou não independentes (associadas); já na regressão logística multivariada as relações são avaliadas à luz de todas as variáveis presentes no modelo. Em virtude da ausência de dados de alguns pesquisadores optou-se por trabalhar, excepcionalmente, com um nível de significância de $15 \%{ }^{3}$.

Finalmente, no texto que se segue, serão discutidas e problematizadas cinco hipóteses levantadas em consonância com a revisão da literatura:

- As mulheres são maioria em áreas ditas femininas e minoria naquelas ditas masculinas;

- As mulheres são mais qualificadas que os homens;

- Há baixa representação feminina em cargosdaalta hierarquia organizacional;

- Há diferenciais de gênero e geração na produção bibliográfica;

- No caso das mulheres, maternidade e conjugalidade tem impacto negativo sobre a carreira e produção bibliográfica.

3 Em estudo anterior (Oliveira \& Serra, 2014), utilizou-se um nível de significância de 10\% porque foi possível analisar o banco de dados sociodemográfico completo: 712 pesquisadores. Nesse, ainda não constavam dados de produção bibliográfica. Ao concatenar os dados sociodemográficos e de produção bibliográfica, houve a "perda" de pesquisadores/as por falta de dados. 


\section{Resultados e discussão}

\section{Aspectos sociodemográficos}

As/os pesquisadoras/es estão lotadas/os em 6 institutos de pesquisa e 15 de polos de pesquisa científica e tecnológica do estado de São Paulo, no setor do agronegócio. Cada instituto tem uma área de atuação específica, a saber: ciências agrárias, ciências biológicas, ciência de alimentos, recursos pesqueiros e engenharia de pesca, engenharia agrícola e zootecnia; já os polos têm abordagem multidisciplinar.

Nas instituições investigadas, as pesquisadoras constituem branda maioria, pois representam $51,6 \%(\mathrm{~N}=359)$ da população total $(\mathrm{N}=696)$. Neste contexto, foram identificados cenários de predominância masculina e outros de predominância feminina. As mulheres estão mais concentradas nas instituições voltadas à ciência de alimentos, a qual há carreiras predominantemente femininas, como engenharia de alimentos e biologia; enquanto os homens encontram-se majoritariamente nas ciências agrárias, as quais são carreiras predominantemente masculinas a engenharia agrícola e/ou agronômica.

De modo geral, a idade média é de 50,13 anos, sendo que os homens possuem idade média acima da média geral (51,18 anos) e as mulheres abaixo (49,14 anos). As mulheres estão concentradas nos grupos de idade mais jovens, enquanto os homens nos grupos mais avançados. Agregando os percentuais num grande grupo etário de 54 anos ou menos, observou-se que o percentual de mulheres é de $36,8 \%$, enquanto o de homens de $28,6 \%$. No grupo etário de 55 anos ou mais, elas representam 14,8\%, enquanto eles $19,8 \%$.

Do total de mulheres, 40,4\% prestam serviços há 8 anos ou menos e $28,1 \%$ há 26 anos ou mais. Os homens estão bem distribuídos nos três grupos de tempo de admissão: 34,7\% ( $\leq 8$ anos), 30,9\% (9-25 anos) e 34,4\% ( $\geq 26$ anos).

As datas deadmissão revelaram queas mulheres entraram de forma mais massiva nas instituições a partir da década de 1980, conforme pode ser observado na Figura 1. Isto pode justificar o fato de serem mais jovens que os homens e, em sua maioria, estarem prestando serviços há menos tempo. Neste sentido, houve um processo de "feminilização"4 da carreira de "pesquisador científico" nas instituições. Soma-se a isso o maior acesso à formação acadêmica e o crescente estímulo à desconstrução de gênero nas ciências.

4 Yannoulas (2011) apresenta noções diferenciadas sobre feminilização e feminização. Para a autora, a feminilização possui um significado quantitativo e refere-se ao aumento do peso relativo do sexo feminino na composição de uma profissão ou ocupação; já a feminização tem um significado qualitativo que alude às transformações de significado e valor social de uma profissão ou ocupação, originadas a partir da feminilização ou aumento quantitativo e vinculadas à concepção de gênero predominante em uma época. 
Figura 1: Admissão das/os pesquisadoras/es nas instituições (1969-2013).

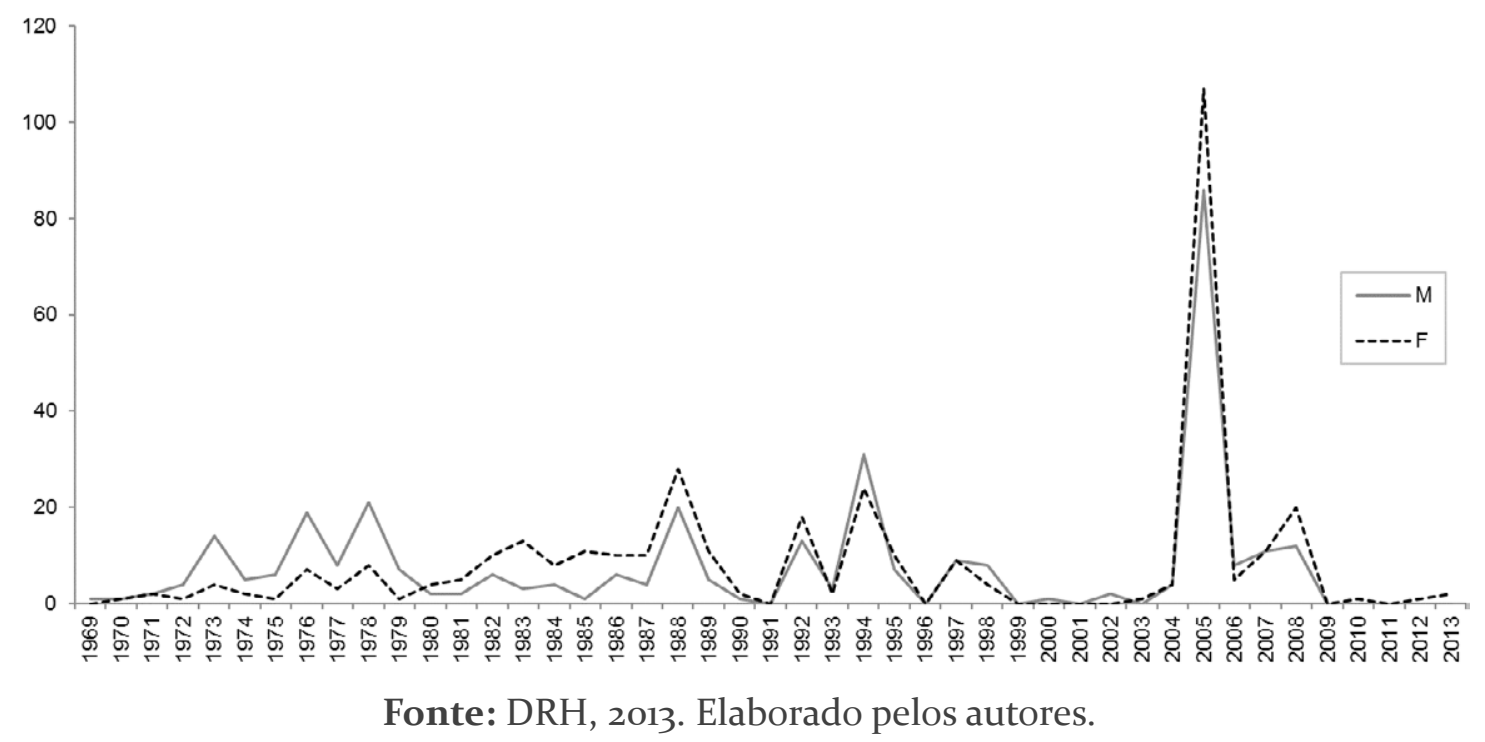

Com relação à escolaridade, as mulheres são levemente mais escolarizadas que os homens, pois $59,9 \%$ delas possuem doutorado, contra $57,9 \%$ dos pesquisadores do sexo masculino. Em relação ao pós-doutorado, 13,65\% delas possuem esta titulação, enquanto os pesquisadores $12,76 \%$. Na titulação mais baixa (graduação), eles são maioria (6,23\%; elas: 4,46\%). Quando cruzados os dados de escolaridade com os grupos etários ( $\leq 44$ anos; 45 a 54 anos; $\geq 55$ anos) constatou-se que, para ambos os sexos, os mais jovens são mais escolarizados que os mais envelhecidos. Podem explicar o fenômeno: a expansão do acesso à universidade nas últimas décadas e a crescente exigência de títulos para a ascensão profissional.

No que diz respeito à nupcialidade, $62,7 \%$ dos homens estão em união, contra $56,8 \%$ das mulheres. Outro dado relevante é que cerca de um terço das pesquisadoras estão solteiras (34\%). E quanto mais envelhecida a mulher, menor o contingente daquelas que estão em união.

O número médio de filhos da população é 1,01. Os homens $(1,08)$ possuem uma média maior que a das mulheres $(0,94)$. Para ambos os sexos, o número máximo de filhos é 5 . Cerca de 41,4\% dos pesquisadores não possuem filhos, sendo que no caso feminino esse percentual sobe para $42,3 \%$. Quanto mais jovem, menor é a média de filhos. Com relação a esta temática, cabe mencionar que nas últimas décadas houve a queda de dois indicadores demográficos relacionados à mulher: as taxas de nupcialidade e fecundidade. Tem sido observado o aumento na incidência de divórcio, bem como de recasamentos. Neste sentido, houve a influência da escolaridade e do mercado de trabalho sobre tais índices (Berquó \& Oliveira, 1992; Berquó \& Cavenaghi, 2006).

Nas instituições, existe a possibilidade de receber promoções na carreira ao longo do tempo e ascender níveis no cargo. O nível do cargo é expresso em números romanos, de modo crescente: I, II, III, IV, V e VI. De modo geral, há certa equidade entre homens e mulheres, com branda maioria feminina nos níveis mais altos (V e VI).

Por fim, os pesquisadores podem vir a ocupar cargos de direção nas instituições de pesquisa. Com relação à população total, 19,1\% ocupam alguma diretoria, sendo 
que desses apenas 7,5\% ocupam os três cargos mais altos. Nesses, as mulheres são minoria. Tal fenômeno será abordado na última seção de análise dos dados.

\section{Produção bibliográfica}

O volume total acumulado de produção bibliográfica no período analisado (1969-2013) foi de 70.884 trabalhos publicados. As mulheres (34.120) e os homens (36.764) possuem volumes próximos de produção bibliográfica acumulada total. Segundo ilustra a Figura 2, os homens possuem maior volume de livros, capítulos, textos e artigos; já as mulheres, maior volume de anais de congressos.

Neste sentido, alguns estudos de localidades distintas (Larivière et al., 2011; Leta, 2014) sugerem que as mulheres têm menores índices de colaboração que os homens, o que pode explicar essa ligeira desvantagem em publicações para além dos anais de congressos. Embora haja um desempenho geral masculino superior, existem diferenças entre áreas em que as mulheres podem demonstrar produtividade mais elevada (Leta, 2014).

Figura 2: Produção bibliográfica acumulada (1969-2013) das/os pesquisadoras/es, por tipo de publicação.

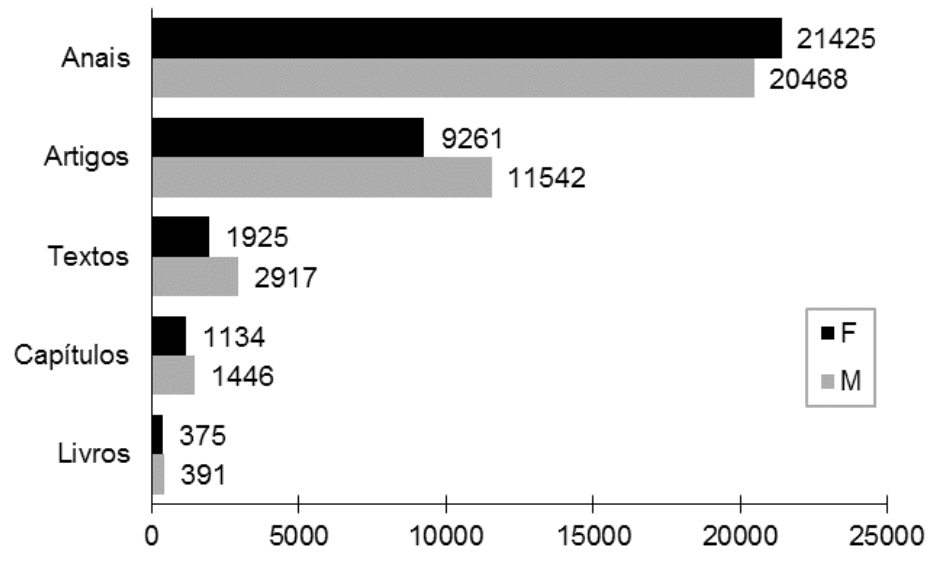

Fonte: Plataforma Lattes (CNPq), 2016; DRH, 2013. Elaborado pelos autores.

Os dados evidenciaram ainda diferenças geracionais na produção bibliográfica de homens e mulheres, conforme pode ser observado na Figura 3. Nas gerações mais envelhecidas ( $\geq 55$ anos), a média de publicações masculina $(\bar{x}=117,4)$ é muito superior à feminina $(\bar{x}=102,3)$. No entanto, essa divergência se reduz na geração mais jovem ( $\leq 44$ anos), pois as mulheres $(\bar{x}=78)$ demonstram certa paridade na média da produção bibliográfica total em relação aos homens $(\bar{x}=82,7)$. Em outras palavras, os dados parecem apontar que as tradicionais diferenças de desempenho entre homens e mulheres tendem a desaparecer com o tempo. Esses resultados vão de encontro aos achados de Arensbergen et al. (2011).

Ainda sobre os grupos etários, as pesquisadoras concentram a sua maior produção bibliográfica na faixa dos 45 a 54 anos (41,9\%). Os pesquisadores, por outro 
lado, concentram o maior volume de produção bibliográfica no grupo de 55 anos ou mais (44,1\%). Isto se justifica quando observado a maior concentração da população masculina na faixa etária dos 55 anos ou mais $(19,8 \%)$ e a feminina na faixa de 45 a 54 anos $(19,7 \%)$. Outro fator a levar em consideração é o resultado da análise quiquadrado que indica uma associação entre produtividade e idade ( $\mathrm{p}=\mathrm{o}, \mathrm{oooo})$, isto é, quanto mais quanto mais velho/a o/a pesquisador/a maior o número de produção total.

Figura 3: Produção bibliográfica acumulada (1969-2013) dos/as pesquisadores/as, segundo grupos de idade.

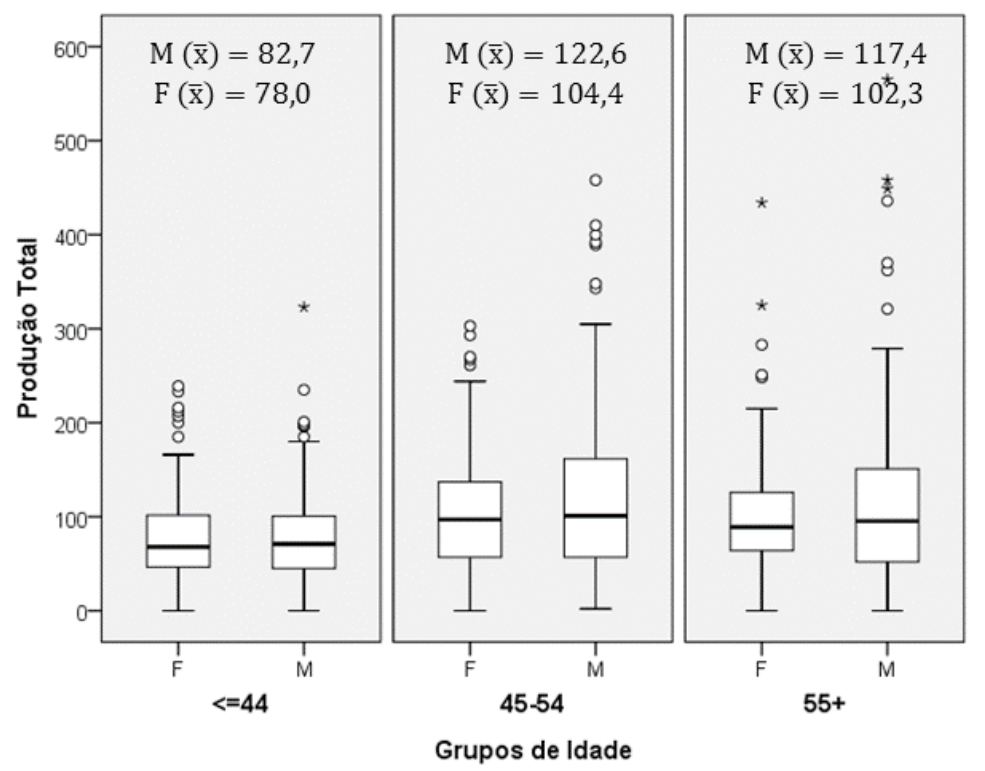

Fonte: Plataforma Lattes (CNPq), 2016; DRH, 2013. Elaborado pelos autores.

De acordo com a Figura 4, pode ser observado que, embora as mulheres apresentem volume acumulado total de produção bibliográfica ligeiramente inferior ao dos homens, elas possuem um maior incremento médio de publicação anual. Além disso, outros dois fatores chamam atenção: acentuadas quedas da produção bibliográfica de ambos os sexos ao longo dos anos e o alto incremento de produção bibliográfica feminina na década de 8o. Tamanho incremento de publicações pode ser explicado pela intensa entrada de pesquisadoras nas instituições na década de 1980, o que gerou saldo positivo na produção científica institucional. Já a queda na produtividade pode ser apreendida pela acentuada evasão de pesquisadores/as ao longo dos anos. 
Figura 4: Taxa média geométrica de crescimento anual da produção bibliográfica, segundo sexo (1975-2009)

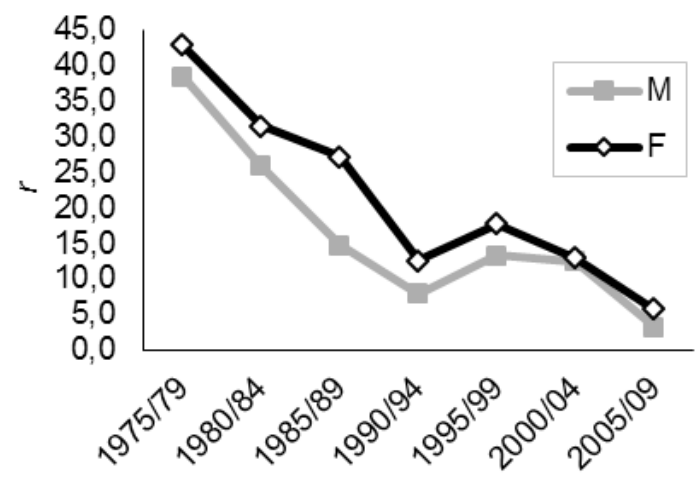

Fonte: Plataforma Lattes (CNPq), 2016; DRH, 2013. Elaborado pelos autores.

Assim como Leta (2014), esta seção confrontou a máxima de que a produção bibliográfica masculina é superior à feminina. Através de gráficos e indicadores, os resultados assinalaram ressalvas. Ainda que haja produtividade masculina superior à feminina no geral, quando desagregados os dados por tipo de publicação e grupos de idade diferenças emergiram. As mulheres são mais produtivas que os homens na publicação de Anais de Congressos, por exemplo. Outros dois fenômenos interessantes dizem respeito ao fato de as mulheres mais jovens possuírem paridade na produção bibliográfica acumulada em relação aos homens da mesma faixa etária e maior incremento médio de publicação anual. Esses achados realçam uma tendência de paridade ou reversão das mulheres na produtividade cientifica, o que pode ser justificado pela sua maior escolaridade em relação aos homens, principalmente em se tratando das mulheres mais jovens.

\section{Pesquisadoras e o desenvolvimento da carreira}

Na análise estatística multivariada, observou-se que o sexo $(\mathrm{p}=0,1373)$ é uma variável importante para determinar a ocupação dos altos cargos de direção. E quanto mais alto é o cargo, menor é a penetração feminina. Nos dois mais altos cargos de diretoria, as mulheres representam $25 \%$ do total. Os homens têm 1,62 vezes mais chance de estar em altos cargos que as mulheres. No entanto, as pesquisadoras possuem maior qualificação acadêmica, estão em níveis mais altos na carreira de pesquisa e possuem incremento médio de publicação anual superior ao dos homens. Sendo assim, os argumentos da qualificação e produtividade são insuficientes para explicar o fenômeno.

Ainda que tenham adentrado nas instituições, as configurações de gênero presentes no corpus social e organizacional complexificam o desenvolvimento da carreira das pesquisadoras. Na literatura, há uma abordagem que busca compreender as barreiras de gênero na carreira das mulheres, conhecida como glass ceiling (teto 
de vidro). Esse enfoque refere-se às discriminações não explícitas que impedem o acesso das mulheres aos altos níveis hierárquicos das organizações de trabalho, postos e ocupações associados à melhor status e rendimento. A sua extensão teórica procura explicar as motivações para as barreiras invisíveis e dificilmente transponíveis para a ascensão profissional feminina (Madalozzo, 2010).

A maternidade como um empecilho na progressão da carreira feminina tem sido problematizada nos estudos de gênero. Neste caso, os resultados dos testes quiquadrado revelaram associação significativa $(\mathrm{p}=\mathrm{0}, 0154)$ entre produção bibliográfica e número de filhos. Em outras palavras, quanto maior o número de filhos, maior é a produtividade das pesquisadoras. Na regressão multivariada, foi observado que as pesquisadoras com um filho ( $\mathrm{p}=0,0366$ ) possuem 4,78 vezes mais chances de ocupar um alto cargo que aquelas sem filhos. Já aquelas com dois ou mais filhos ( $\mathrm{p}=0,0335)$ possuem 4,47 vezes mais chances. Os achados contrapõem estudos, como o de Bruschini \& Puppin (2004), que apresentam o fato de a mulher ter filhos como um limitador para ascensão a altos cargos.

Fox (2005) analisou a relação entre nupcialidade, maternidade e produção científica de mulheres cientistas, em comparação aos homens. Observou que mulheres no segundo casamento ou casadas com um cientista tiveram maior produtividade que aquelas no primeiro casamento; já mulheres cientistas com filho na pré-escola se mostraram mais produtivas que aquelas sem filho ou com filhos na escola. Esses resultados indicam o quão complexa é a apreensão da relação entre nupcialidade/ maternidade e o desempenho científico das mulheres.

As estatísticas apresentadas apontam que, se por um lado, as mulheres têm participado cada vez mais das atividades de C\&T no Brasil, por outro, elas pouco avançam nas mais altas posições gerenciais - achados que vão de encontro àqueles evidenciados por Leta (2003). Logo, olhar estritamente para a qualificação, maternidade e produtividade se apresenta como insuficiente para explicar esse fenômeno.

\section{Conclusões}

Este artigo abordou mulheres de diferentes tempos e a conquista por espaços na pesquisa agropecuária paulista. Neste sentido, teve como objetivo principal verificar a relação entre atributos sociodemográficos e o desenvolvimento da carreira de pesquisadoras lotadas em instituições públicas de pesquisa paulistas, no contexto do agronegócio. Os objetivos (geral e específicos) propostos foram alcançados e as hipóteses postas em suspensão na medida em que descrevemos a participação, contribuição e inserção feminina em um campo onde as mulheres são historicamente sub-representadas: a ciência agropecuária. Na relação com a abordagem de gênero e geração nas ciências, articulamos problematizações e sugerimos explicações sobre os resultados encontrados. 
Em suma, verificamos que as pesquisadoras: i) são grande maioria nas instituições de áreas ditas femininas, como as ciências biológicas e ciências de alimentos, e minoria nas instituições de ciências ditas masculinas, principalmente, as ciências agrárias; ii) entraram nas instituições de forma mais acentuada a partir da década de 1980 ("feminilização"); iii) são mais jovens que os homens; iv) estão há menos tempo prestando serviços nas instituições; v) são ligeiramente mais escolarizadas que os pesquisadores, principalmente nos grupos etários mais jovens; vi) estão em sua grande maioria em união, sendo que cerca de um terço estão solteiras; vii) quanto mais envelhecidas, menor o contingente daquelas que estão em união; viii) possuem um número médio de filhos mais baixo que os homens; ix) quanto mais jovens, mais acentuada é a queda da média de filhos; $x$ ) são branda maioria nos níveis mais altos do cargo de pesquisador científico; xi) e expressiva minoria nos mais altos cargos de direção.

No que tange à produção bibliográfica, as pesquisadoras: i) apesar de apresentarem volume acumulado total levemente menor que os homens, possuem um maior incremento médio de publicação anual; ii) concentram a sua maior produção bibliográfica nas faixas etárias mais jovens; iii) mais jovens possuem paridade na produção bibliográfica acumulada em relação aos homens da mesma faixa etária, indicando um horizonte de mudanças; iv) e quanto maior o número de filhos, maior a produção bibliográfica.

A partir do exposto, observamos a complexidade do fenômeno, no qual a tríplice linha argumentativa qualificação-maternidade-produtividade mostrou-se insuficiente para explicar a baixa penetração feminina nos mais altos cargos de direção. Na realidade investigada, a reflexão sobre a assimetria de gênero sob interpretação simbólica (Bourdieu, 2002) pode oferecer possibilidades de compreensão, sendo a perspectiva do "teto de vidro" capaz de lançar luz direcionada à questão. Conforme nos lembra Leta (2014), a discriminação de gênero nas instituições de pesquisa possui caráter multifacetado. Trata-se, portanto, de um desafio a ser enfrentado não apenas por mulheres cientistas, mas, sobretudo, pelos homens.

\section{Referências}

ARENSBERGEN, Pleun. et al. (2012). "Gender differences in scientific productivity: a persisting phenomenon?”. Scientometrics, n. 3, v. 93: p. 857-868, dec.

BERQUÓ, Elza \& OLIVEIRA, Maria Coleta. (1992). "Casamento em tempos de crise". Revista Brasileira de Estudos de População, n. 2, v. 9: p. 155-167.

\& CAVENAGHI, Suzana. (2006). "Breve nota sobre a redução no número médio de filhos por mulher no Brasil”. Novos estudos - CEBRAP, n. 74: p.11-15, mar. 
BOURDIEU, Pierre. (2002). A dominação masculina. 2a edição. Rio de Janeiro: Bertrand Brasil.

BRASIL. (2011). Agropecuária é o setor com maior crescimento na última década. Disponível em: <http://www.brasil.gov.br/noticias/arquivos/2011/o3/4/agropecuariae-o-setor-com-maior-crescimento-na-ultima-decada >. Acesso em: 21 mai. 2013.

BRUSCHINI, Cristina \& PUPPIN, Andrea (2004). "Trabalho de mulheres executivas no Brasil no final do século XX”. Cadernos de Pesquisa, v. 34, n. 121: p.105-138, jan-abr.

CARVALHO,MariaEulinaetal.(2013)."Carreirasdocentesdemulheresemdepartamentos masculinos: mudanças geracionais”. In: SEMINÁRIO INTERNACIONAL FAZENDO GÊNERO, 10, Florianópolis. Anais eletrônicos... Florianópolis: UFSC. Disponível em: <http://www.fg2013.wwc2017.eventos.dype.com.br/resources/anais/20/1373313829_ ARQUIVO_CarvalhoRabaySilvaFazendoGenero2013.pdf>. Acesso em: 14 jul. 2017.

CERQUEIRA, Cézar \& GIVISIEZ, Gustavo. (2004). "Conceitos básicos em demografia e dinâmica demográfica brasileira”. In: E. Rios-Neto \& Ruas-Riani (orgs.). Introdução à demografia da educação,Campinas,ABEP.

CGEE, Centro de Gestão e Estudos Estratégicos. (2012). Mestres 2012: estudos da demografia da base técnico-científica brasileira. Brasília: CGEE. Disponível em: <http://www.cgee.org. br/publicacoes/mestres_e_doutores.php>.Acesso em: 21 mai. 2013.

COSTA, Maria Conceição \& FELTRIN, Rebeca. (2016). "Desafios da interseccionalidade em gênero, ciência e tecnologia”. Cadernos Pagu, n. 47.

FOX, Mary Frank. (2005). "Gender, family characteristics, and publication productivity among scientists". Social Studies of Science, n. 35, v. 1: p.131-150.

IBGE, Instituto Brasileiro de Geografia e Estatística. (2012). Censo Demográfico 2010: Trabalho e rendimento - resultados da amostra. Rio de Janeiro: IBGE. Disponível em: <http://www.ibge.gov.br/home/estatistica/populacao/censo2010/trabalho_e_ rendimento/default.shtm>. Acesso em: 16 abr. 2013.

KELLER, Evelyn Fox. (2006). “Qual foi o impacto do feminismo na ciência?”. Cadernos Pagu, n. 27: p.13-34, jul-dez.

LARIVIÈRE, Vincent. et. al. (2011). "Sex differences in research funding, productivity and impact: an analysis of Quèbec university professors". Scientometricsn. 3, v. 87: p.483-498, jun. 
LETA, Jacqueline. (2003). "As mulheres na ciência brasileira: crescimento, contrastes e um perfil de sucesso”. Revista de Estudos Avançadosn. 49, v. 17: p.271-284.

et al. (2006). "As mulheres na pesquisa, no desenvolvimento tecnológico e na inovação: uma comparação Brasil/França”. Revista do Serviço Público, n. 4, v. 57:p.531547, out-dez.

. (2014). “Mulheres na Ciência Brasileira: desempenho inferior?". Revista Feminismos, n. 3, v. 2: p.139-152, set-dez.

MADALOZZO, Regina. (2010). "Teto de vidro e identificação: uma análise do perfil de CEOs no Brasil”. Insper Working Paper. Disponível em: <http://www.insper.edu.br/ working-papers/working-papers-2010/teto-de-vidro-e-identificacao-uma-analisedo-perfil-de-ceos-no-brasil/>. Acesso em: 22 mai. 2013.

MELO, Hildete \& OLIVEIRA, André. (2006). "A produção científica brasileira no feminino". Cadernos Pagu, n. 27: p.301-331.

MOREIRA, Maria Lígia \& VELHO, Lea. (2010). "Pós-graduação do Instituto Nacional de Pesquisas Espaciais numa perspectiva de gênero". Cadernos Pagu, n. 35: p.279-3o8, juldez.

OLINTO, Gilda. (2011). "A inclusão das mulheres nas carreiras de ciência e tecnologia no Brasil”. Inclusão Social, v. 5: p.68-77, jul-dez.

OLIVEIRA, Kris \& SERRA, Márcia. (2014). “A demografia organizacional de instituições de pesquisa paulistas - uma abordagem de gênero". In: XIX ENCONTRO NACIONAL DE ESTUDOS POPULACIONAIS, São Pedro. Anais eletrônicos... São Pedro: ABEP. Disponível em: < http://www.abep.org.br/publicacoes/index.php/anais/article/ view/2148/2103>. Acesso em: 14 jul. 2017.

OLIVEIRA, Maria Aparecida et al. (1999). "Comprometimento no trabalho e produção científica entre pesquisadores brasileiros”. Revista de Administração, n. 3, v. 34: p.1220, jul-set.

PFEFFER, Jeffrey. (1985). “Organizational demography: implications for management”. California Management Review, v. 28: p.67-81.

PLATAFORMA LATTES - CNPq, Conselho Nacional de Desenvolvimento Científico e Tecnológico. (2016). Buscar Currículo Lattes. Disponível em: <http://buscatextual.cnpq. br/buscatextual/busca.do>. Acesso em: Acesso em: 15 dez. 2016. 
PRADO, Renata \& FLEITH, Denise. (2012). "Pesquisadoras brasileiras: conciliando talento, ciência e família”. Arquivos Brasileiros de Psicologia, n. 64, v. 2, p.19-34.

RIGOLIN, Camila et al. (2013). "Métricas da participação feminina na ciência e na tecnologia no contexto dos INCTs: primeiras aproximações". Liinc em Revista, v. 9: p.143-170.

RODRIGUES, Jeorgina \& GUIMARÃES, Maria Cristina. (2016). "A Fundação Oswaldo Cruz e a ciência no feminino: a participação feminina na prática e na gestão da pesquisa em uma instituição de ensino e pesquisa”. Cadernos Pagu, n. 46: p.197-222.

SABOYA, Maria Clara. (2013). "Relações de gênero, ciência e tecnologia: uma revisão da bibliografia nacional e internacional”. Educação, Gestão e Sociedade, n. 12, v. 3: p.126.

SCOTT, Joan. (1995). "Gênero: uma categoria útil de análise histórica”. Educação E Realidade, n. 2, v. 20, p.71-99, jul.-dez.

VELHO, Lea \& LEÓN, Elena. (1998). "A construção social da produção científica por mulheres". Cadernos Pagu, n. 10: p.309-344.

YANNOULAS, Silvia. (2011). "Feminização ou Feminilização? Apontamentos em torno de uma categoria”. Temporalis, n. 22, v. 11, p.271-292, jul-dez.

Recebido: 20.09.2017

Aceito: 30.05 .2018 